\title{
Four Mappings Satisfying $\Psi$-Contractive Type Condition and Having Unique Common Fixed Point on 2-Metric Spaces*
}

\author{
Hailan Jin, Yongjie Piao \\ Department of Mathematics, College of Science, Yanbian University, Yanji, China \\ Email: hljin98@ybu.edu.cn,pyj6216@hotmail.com
}

Received November 7, 2012; revised January 21, 2013; accepted January 30, 2013

\begin{abstract}
In this paper, we introduce a class $\Psi$ of real functions defined on the set of non-negative real numbers, and obtain a new unique common fixed point theorem for four mappings satisfying $\Psi$-contractive condition on a non-complete 2-metric space and give the versions of the corresponding result for two and three mappings.
\end{abstract}

Keywords: 2-Metric Space; Class $\Psi$; Cauchy Sequence; Coincidence Point; Common Fixed Point

\section{Introduction and Preliminaries}

Using subsidiary conditions [1,2] such as commutability of mappings or uniform boundless of mappings at some point and so on, many authors have discussed and obtained many unique common fixed point theorems of mappings with some contractive or quasi-contractive condition on 2-metric spaces. The author [3-7] obtained similar results for infinite mappings with contractive conditions or quasicontractive conditions under removing the above subsidiary conditions. These results generalized and improved many same type unique common fixed point theorems. Recently, the author [8] discussed the existence of coincidence points and common fixed points for four mappings with $\Phi$-contractive conditions on 2-metric spaces and give some corresponding results.

Here, by introducing a new class $\Psi$ of real functions defined on $[0,+\infty)$, we will discuss the existence problem of unique common fixed points for four mappings with $\psi$-contractive type condition on non-complete 2-metric spaces and give some corresponding forms.

The following definitions and lemmas are well known.

Definition1.1. ([3]) A 2-metric space $(X, d)$ consists of a nonempty set $X$ and a function

$d: X \times X \times X \rightarrow[0,+\infty)$ such that

1) For distant elements $x, y \in X$, there exists an $u \in X$ such that $d(x, y, u) \neq 0$;

2) $d(x, y, z)=0$ if and only if at least two elements in $\{x, y, z\}$ are equal;

3) $d(x, y, z)=d(u, v, w)$, where $\{u, v, w\}$ is any permutation of $\{x, y, z\}$;

*Project supported by NNSFC (No. 11261062).
4) $d(x, y, z) \leq d(x, y, u)+d(x, u, z)+d(u, y, z)$ for all $x, y, z, u \in X$.

Definition 1.2. ([3]) A sequence $\left\{x_{n}\right\}_{n \in \mathbb{N}}$ in 2-metric space $(X, d)$ is said to be Cauchy sequence, if for each $\varepsilon>0$ there exists a positive integer $N \in \mathbb{N}$ such that $d\left(x_{n}, x_{m}, a\right)<\varepsilon$ for all $a \in X$ and $n, m>N$.

Definition 1.3. ([3,4]) A sequence $\left\{x_{n}\right\}_{n \in \mathbb{N}}$ is said to be convergent to $x \in X$, if for each $a \in X$, $\lim _{n \rightarrow+\infty} d\left(x_{n}, x, a\right)=0$. And we write that $x_{n} \rightarrow x$ and call $x$ the limit of $\left\{x_{n}\right\}_{n \in \mathbb{N}}$.

Definition 1.4. $([3,4])$ A 2-metric space $(X, d)$ is said to be complete, if every Cauchy sequence in $X$ is convergent.

Definition 1.5. ([9,10]) Let $f$ and $g$ be self-maps on a set $X$. If $w=f x=g x$ for some $x \in X$, then $x$ is called a coincidence point of $f$ and $g$, and $w$ is called a point of coincidence of $f$ and $g$.

Definition 1.6. ([11]) Two mappings $f, g: X \rightarrow X$ are weakly compatible, if for every $x \in X$ holds $f g x=g f x$ whenever $f x=g x$.

Lemma 1.7. ([5-7]) Let $(X, d)$ be a 2-metric space and $\left\{x_{n}\right\}_{n \in \mathbb{N}}$ a sequence. If there exists $h \in[0,1)$ such that $d\left(x_{n+2}, x_{n+1}, a\right) \leq h d\left(x_{n+1}, x_{n}, a\right)$ for all $a \in X$ and $n \in \mathbb{N}$, then $d\left(x_{n}, x_{m}, x_{l}\right)=0$ for all $n, m, l \in \mathbb{N}$, and $\left\{x_{n}\right\}_{n \in \mathbb{N}}$ is a Cauchy sequence.

Lemma 1.8. ([5-7]) If $(X, d)$ is a 2-metric space and sequence $\left\{x_{n}\right\}_{n \in \mathbb{N}} \rightarrow x \in X$, then $\lim _{n \rightarrow+\infty} d\left(x_{n}, b, c\right)=d(x, b, c)$ for each $b, c \in X$.

Lemma 1.9. ([9,10]) Let $f, g: X \rightarrow X$ be weakly compatible. If $\{f, g\}$ have a unique point of coincidence $w=f x=g x$, then $w$ is the unique common fixed point of $\{f, g\}$. 


\section{Main Results}

Denoted by $\Psi$ the set of functions $\psi:[0,+\infty) \rightarrow[0,+\infty)$ satisfying the following: $(C 1) \psi$ is continuous and non-decreasing, (C2) $\psi(t)<t$ for all $t>0$.

Remark $\phi \in \Phi$ if and only if $\phi:[0,+\infty)^{5} \rightarrow[0,+\infty)$ is continuous and increasing in each coordinate variable and satisfy that $\phi(t, t, t, 2 t, t)<t$ and $\phi(t, t, t, t, 2 t)<0$ for all $t>0$, see [8]. Obviously, the set $\Psi$ is vary different from the set $\Phi$.

Example Let $\psi_{1}, \psi_{2}:[0,+\infty) \rightarrow[0,+\infty)$ be defined by $\psi_{1}(t)=k t, 0<k<1$

$$
\psi_{2}(t)= \begin{cases}\frac{t}{2}, & \text { for } 0 \leq t \leq 1 \\ t-\frac{1}{2}, & \text { for } t>1 .\end{cases}
$$

Then, obviously, $\psi_{1}, \psi_{2} \in \Psi$.

The following is the main result in this paper.

Theorem 2.1. Let $(X, d)$ be a 2-metric space, $S, T, I, J: X \rightarrow X$ four single valued mappings satisfying that $S(X) \subset I(X)$ and $T(X) \subset J(X)$. Suppose that for each $x, y \in X$,

$$
d(S x, T y, a) \leq q \psi\left(\max \left\{d(J x, I y, a), d(J x, S x, a), d(I y, T y, a), \frac{d(J x, T y, a)}{2}, \frac{d(I y, S x, a)}{2}\right\}\right), \quad \forall a \in X,
$$

where $0<q<1$ and $\psi \in \Psi$.

If one of $S(X), T(X), I(X)$ and $J(X)$ is complete, then $T$ and $I, S$ and $J$ have an unique point of coincidence in $X$. Further, $\{I, T\}$ and $\{S, J\}$ are weakly compatible respectively, then $S, T, I, J$ have an unique common fixed point in $X$.

Proof Take any element $x_{0} \in X$, then in view of the conditions $S(X) \subset I(X)$ and $T(X) \subset J(X)$, we can construct two sequences $\left\{x_{n}\right\}$ and $\left\{y_{n}\right\}$ as follows:

$$
y_{2 n}=S x_{2 n}=I x_{2 n+1}, y_{2 n+1}=T x_{2 n+1}=J x_{2 n+2}, \forall n=0,1, \cdots \text {. }
$$

For any fixed $n=0,1, \cdots$, by (1) and $(C 1)$ and (iv) in definition 1.1, we obtain that

$$
\begin{aligned}
& d\left(y_{2 n}, y_{2 n+1}, a\right)=d\left(S x_{2 n}, T x_{2 n+1}, a\right) \\
& \leq q \psi\left(\max \left\{d\left(J x_{2 n}, I x_{2 n+1}, a\right), d\left(J x_{2 n}, S x_{2 n}, a\right), d\left(I x_{2 n+1}, T x_{2 n+1}, a\right), \frac{d\left(J x_{2 n}, T x_{2 n+1}, a\right)}{2}, \frac{d\left(I x_{2 n+1}, S x_{2 n}, a\right)}{2}\right\}\right) \\
& =q \psi\left(\max \left\{d\left(y_{2 n-1}, y_{2 n}, a\right), d\left(y_{2 n}, y_{2 n+1}, a\right), \frac{d\left(y_{2 n-1}, y_{2 n+1}, a\right)}{2}\right\}\right) \\
& \leq q \psi\left(\max \left\{d\left(y_{2 n-1}, y_{2 n}, a\right), d\left(y_{2 n}, y_{2 n+1}, a\right), \frac{d\left(y_{2 n-1}, y_{2 n}, y_{2 n+1}\right)+d\left(y_{2 n-1}, y_{2 n}, a\right)+d\left(y_{2 n}, y_{2 n+1}, a\right)}{2}\right\}\right) .
\end{aligned}
$$

Suppose that $d\left(y_{2 n-1}, y_{2 n}, y_{2 n+1}\right)>0$.

Take $x=x_{2 n}, y=x_{2 n+1}, a=y_{2 n-1}$, then by (1) and definition 1.1 and $(C 2)$, we obtain that

$$
\begin{aligned}
& d\left(y_{2 n-1}, y_{2 n}, y_{2 n+1}\right)=d\left(S x_{2 n}, T x_{2 n+1}, y_{2 n-1}\right) \\
& \leq q \psi\left(\operatorname { m a x } \left\{d\left(J x_{2 n}, I x_{2 n+1}, y_{2 n-1}\right), d\left(J x_{2 n}, S x_{2 n}, y_{2 n-1}\right),\right.\right. \\
& \left.\left.\quad d\left(I x_{2 n+1}, T x_{2 n+1}, y_{2 n-1}\right), \frac{d\left(J x_{2 n}, T x_{2 n+1}, y_{2 n-1}\right)}{2}, \frac{d\left(I x_{2 n+1}, S x_{2 n}, y_{2 n-1}\right)}{2}\right\}\right) \\
& =q \psi\left(d\left(y_{2 n-1}, y_{2 n}, y_{2 n+1}\right)\right)<q d\left(y_{2 n-1}, y_{2 n}, y_{2 n+1}\right),
\end{aligned}
$$

which is a contradiction since $0<q<1$.

Hence $d\left(y_{2 n-1}, y_{2 n}, y_{2 n+1}\right)=0$, so we have that

$$
\begin{aligned}
& d\left(y_{2 n}, y_{2 n+1}, a\right)=d\left(S x_{2 n}, T x_{2 n+1}, a\right) \\
& \leq q \psi\left(\max \left\{d\left(y_{2 n-1}, y_{2 n}, a\right), d\left(y_{2 n}, y_{2 n+1}, a\right), \frac{d\left(y_{2 n-1}, y_{2 n}, a\right)+d\left(y_{2 n}, y_{2 n+1}, a\right)}{2}\right\}\right) .
\end{aligned}
$$


If $d\left(y_{2 n-1}, y_{2 n}, a\right)<d\left(y_{2 n}, y_{2 n+1}, a\right)$ for some $a \in X$, then (2) becomes that

$$
\begin{aligned}
& d\left(y_{2 n}, y_{2 n+1}, a\right) \\
& \leq q \psi\left(d\left(y_{2 n}, y_{2 n+1}, a\right)\right)<q d\left(y_{2 n}, y_{2 n+1}, a\right) .
\end{aligned}
$$

This is a contradiction. Hence for all $a \in X$, so we have that

$$
\begin{aligned}
& d\left(y_{2 n}, y_{2 n+1}, a\right) \\
& \leq q \psi\left(d\left(y_{2 n-1}, y_{2 n}, a\right)\right) \leq q d\left(y_{2 n-1}, y_{2 n}, a\right) .
\end{aligned}
$$

Similarly, we can obtain that

$$
d\left(y_{n+1}, y_{n+2}, a\right) \leq q d\left(y_{n}, y_{n+1}, a\right) .
$$

Hence we have that

$$
\begin{aligned}
& d(u, T v, a) \\
& \leq d\left(y_{2 n}, T y, a\right)+d\left(u, y_{2 n}, a\right)+d\left(u, y_{2 n}, T v\right) \\
& =d\left(S x_{2 n}, T v, a\right)+d\left(u, y_{2 n}, a\right)+d\left(u, y_{2 n}, T v\right) \\
& \leq q \psi\left(\operatorname { m a x } \left\{d\left(J x_{2 n}, I v, a\right), d\left(J x_{2 n}, S x_{2 n}, a\right), d(I v, T v, a),\right.\right. \\
& =q \psi\left(\operatorname { m a x } \left\{d\left(y_{2 n-1}, u, a\right), d\left(y_{2 n-1}, y_{2 n}, a\right), d(u, T v, a),\right.\right. \\
& \text { Let } n \rightarrow \infty, \operatorname{then} \text { by }(C 1) \text { and Lemma 1.8, the above } \\
& d(u, T v, a) \leq q \psi\left(\max \left\{d(u, T v, a), \frac{d(u, T v, a)}{2}\right\}\right)
\end{aligned}
$$$$
\leq q \psi\left(\max \left\{d\left(J x_{2 n}, I v, a\right), d\left(J x_{2 n}, S x_{2 n}, a\right), d(I v, T v, a), \frac{d\left(J x_{2 n}, T v, a\right)}{2}, \frac{d\left(I v, S x_{2 n}, a\right)}{2}\right\}\right)+d\left(u, y_{2 n}, a\right)+d\left(u, y_{2 n}, T v\right)
$$$$
=q \psi\left(\max \left\{d\left(y_{2 n-1}, u, a\right), d\left(y_{2 n-1}, y_{2 n}, a\right), d(u, T v, a), \frac{d\left(y_{2 n-1}, T v, a\right)}{2}, \frac{d\left(u, y_{2 n}, a\right)}{2}\right\}\right)+d\left(u, y_{2 n}, a\right)+d\left(u, y_{2 n}, T v\right) \text {. }
$$
that $\left\{y_{2 n+1}\right\} \rightarrow u$.

For any $a \in X$, becomes

If $d(u, T v, a)>0$ for some $a \in X$, then we obtain and $\left\{y_{n}\right\}$ is Cauchy sequence and $\left\{y_{2 n}\right\} \rightarrow u$,we know

$$
d\left(y_{2 n+1}, y_{2 n+2}, a\right) \leq q d\left(y_{2 n}, y_{2 n+1}, a\right), \forall n=0,1, \cdots .
$$

So $\left\{y_{n}\right\}$ is a Cauchy sequence by Lemma 1.7.

Suppose that $I(X)$ is complete, then there exists $u \in I(X)$ and $v \in X$ such that

$y_{2 n}=S x_{2 n}=I x_{2 n+1} \rightarrow u=I v$. (If $S(X)$ is complete, there exists $u \in S(X) \subset I(X)$, then the conclusions remains the same). Since

$$
\begin{aligned}
& d\left(y_{2 n+1}, u, a\right) \\
& \leq d\left(y_{2 n+1}, y_{2 n}, a\right)+d\left(y_{2 n}, u, a\right)+d\left(y_{2 n}, y_{2 n+1}, u\right),
\end{aligned}
$$

from (3) that $d(u, T v, a)<q d(u, T v, a)$, which is a contradiction since $0<q<1$. Hence $d(u, T v, a)=0$ for all $a \in X$, so $T v=u=I v$, i.e., $u$ is a point of coincidence of $T$ and $I$, and $v$ is a coincidence point of $T$ and $I$.

Since $u=T v \in T(X) \subset J(X)$, there exists $w \in X$

$$
\begin{aligned}
& d(S w, u, a) \\
& \leq d\left(S w, y_{2 n+1}, a\right)+d\left(y_{2 n+1}, u, a\right)+d\left(y_{2 n+1}, u, S w\right) \\
& =d\left(S w, T x_{2 n+1}, a\right)+d\left(y_{2 n+1}, u, a\right)+d\left(y_{2 n+1}, u, S w\right) \\
& \leq q \psi\left(\max \left\{d\left(J w, I x_{2 n+1}, a\right), d(J w, S w, a), d\left(I x_{2 n+1}, T x_{2 n+1}, a\right), \frac{d\left(J w, T x_{2 n+1}, a\right)}{2}, \frac{d\left(I x_{2 n+1}, S w, a\right)}{2}\right\}\right) \\
& +d\left(y_{2 n+1}, u, a\right)+d\left(y_{2 n+1}, u, S w\right) \\
& \leq q \psi\left(\max \left\{d\left(u, y_{2 n}, a\right), d(u, S w, a), d\left(y_{2 n}, y_{2 n+1}, a\right), \frac{d\left(u, y_{2 n+1}, a\right)}{2}, \frac{d\left(y_{2 n}, S w, a\right)}{2}\right\}\right)+d\left(y_{2 n+1}, u, a\right)+d\left(y_{2 n+1}, u, S w\right) .
\end{aligned}
$$
such that $u=J w$. For any $a \in X$,

Let $n \rightarrow \infty$, then by $(C 1)$ and Lemma 1.8 , we obtain that

$$
\begin{aligned}
d(S w, u, a) & \leq q \psi\left(\max \left\{d(S w, u, a), \frac{d(S w, u, a)}{2}\right\}\right) \\
& =q \psi(d(S w, u, a)) .
\end{aligned}
$$

If $d(S w, u, a)>0$ for some $a \in X$, then the above becomes that $d(S w, u, a)<q d(S w, u, a)$, which is a contradiction since $0<q<1$, so $d(S w, u, a)=0$ for all $a \in X$. Hence $S w=u=J w$, i.e., $u$ is a point of coincidence of $S$ and $J$, and $w$ is coincidence point of $S$ and $J$. Suppose that $z=S x=J x$ is another point 
of coincidence of $S$ and $J$, then there exists $a \in X$ such that $d(z, u, a)>0$, and we have that

$$
\begin{aligned}
& d(z, u, a)=d(S x, T v, a) \\
& \leq q \psi(\max \{d(J x, I v, a), d(J x, S x, a), d(I v, T v, a), \\
& \left.\left.\quad \frac{d(J x, T v, a)}{2}, \frac{d(I v, S x, a)}{2}\right\}\right) \\
& =q \psi(d(z, u, a))<q d(z, u, a),
\end{aligned}
$$

which is a contradiction. So $d(z, u, a)=0$ for all $a \in X$, hence $z=u$, i.e., $u$ is the unique point of coincidence of $S$ and $J$. Similarly, $u$ is also the unique point of coincidence of $T$ and $I$.

By Lemma 1.9, $u$ is the unique common fixed point of $\{S, J\}$ and $\{T, I\}$ respectively, hence $u$ is the unique common fixed point of $S, T, I, J$.

If $J(X)$ or $T(X)$ is complete, then we can also use similar method to prove the same conclusion. We will omit this part.

Using Theorem 2.1 and $\psi_{2}$ in Example, we will obtain the next particular result.

Theorem 2.2. Let $(X, d)$ be a 2-metric space $S, T, I, J: X \rightarrow X$ four single valued mappings satisfying that $S(X) \subset I(X)$ and $T(X) \subset J(X)$. Suppose that for each $x, y, a \in X$

$$
\begin{aligned}
& d(S x, T y, a) \\
& \leq \begin{cases}\frac{q M(x, y, a)}{2}, & \text { for } 0 \leq M(x, y, a) \leq 1 \\
q\left(M(x, y, a)-\frac{1}{2}\right) & \text { for } M(x, y, a)>1,\end{cases}
\end{aligned}
$$

where $0<q<1$ and

$$
\begin{aligned}
& M(x, y, a) \\
& =\max \{d(J x, I y, a), d(J x, S x, a), d(I y, T y, a), \\
& \left.\quad \frac{d(J x, T y, a)}{2}, \frac{d(I y, S x, a)}{2}\right\} .
\end{aligned}
$$

If one of $S(X), T(X)$ and $J(X)$ is complete, then $T$ and $I, S$ and $J$ have an unique point of coincidence in $X$. Further, $\{I, T\}$ and $\{S, J\}$ are weakly compatible respectively, then $S, T, I, J$ have an unique common fixed point in $X$.

The following two theorems are the contractive and quasi-contractive versions of Theorem 2.1 for two mappings.

Theorem 2.3. Let $(X, d)$ be a 2-metric space,

$S, T: X \rightarrow X$ two mappings satisfying that for each $x, y, a \in X$,

$$
\begin{aligned}
& d(S x, T y, a) \\
& \leq q \psi(\max \{d(x, y, a), d(x, S x, a), d(y, T y, a), \\
& \left.\left.\frac{d(x, T y, a)}{2}, \frac{d(y, S x, a)}{2}\right\}\right),
\end{aligned}
$$

where $0<q<1$ and $\psi \in \Psi$. If one of $S(X)$ and $T(X)$ is complete, then $S$ and $T$ have an unique common fixed point in $X$.

Proof Let $I=J=1_{X}$, then by Theorem 2.1, there exist $u \in X$ such that $u$ is the unique point of coincidence of $S$ and $J$. But obviously $S$ and $J$ are weakly compatible, so $u$ is the unique fixed point of $S$ by Lemma 1.9. Similarly, $u$ is also unique fixed point of $T$, hence $u$ is the unique common fixed point of $S$ and $T$.

Theorem 2.4. Let $(X, d)$ be a complete 2-metric space, $I, J: X \rightarrow X$ two subjective mappings satisfying that for each $x, y, a \in X$,

$$
\begin{aligned}
& d(x, y, a) \\
& \leq q \psi(\max \{d(J x, I y, a), d(J x, x, a), d(I y, y, a), \\
& \left.\left.\quad \frac{d(J x, y, a)}{2}, \frac{d(I y, x, a)}{2}\right\}\right),
\end{aligned}
$$

where $0<q<1$ and $\psi \in \Psi$. Then $I$ and $J$ have an unique fixed point in $X$.

Proof Let $S=T=1_{X}$, then by Theorem 2.1, there exist $u \in X$ such that $u$ is the unique point of coincidence of $S$ and $J$. But obviously $S$ and $J$ are weakly compatible, so $u$ is the unique fixed point of $J$ by Lemma 1.9. Similarly, $u$ is also unique fixed point of $I$, hence $u$ is the unique common fixed point of $I$ and $J$.

Finally we give two coincidence point theorems for three mappings.

Theorem 2.5. Let $(X, d)$ be a 2-metric space, $S, T, I: X \rightarrow X$ three mappings satisfying that $S(X) \cup T(X) \subset I(X)$. Suppose that for each $x, y, a \in X$,

$$
\begin{aligned}
& d(S x, T y, a) \\
& \leq q \psi(\max \{d(I x, I y, a), d(I x, S x, a), d(I y, T y, a), \\
& \left.\left.\frac{d(I x, T y, a)}{2}, \frac{d(I y, S x, a)}{2}\right\}\right),
\end{aligned}
$$

where $0<q<1$ and $\psi \in \Psi$. If one of $S(X), T(X)$ and $I(X)$ is complete, then $T$ and $I, S$ and $I$ have an unique point of coincidence in $X$. Further, $I$ is one to one mapping, then $S, T, I$ have an unique point of coincidence. 
Proof Let $I=J$, then by Theorem 2.1, there exist a unique element $u \in X$ and $v, w \in X$ such that $u=S v=I v$ and $u=T w=I w$, hence $I v=I w$, which implies that $v=w$, so we obtain that $u=S v=I v=T v$. This means that $u$ is point of coincidence of $T, S, I$. If $u^{\prime}$ is also point of coincidence of $T, S, I$, then $u^{\prime}$ is also point of coincidence of $S, I$, hence by uniqueness of points of coincidence of $S$ and $I$, we have that $u=u^{\prime}$. Hence $u$ is the unique point of coincidence of $S, T, I$.

Theorem 2.6. Let $(X, d)$ be a 2-metric space, $S, I, J: X \rightarrow X$ three mappings satisfying that $S(X) \subset I(X) \cap J(X)$. Suppose that for each $x, y, a \in X$,

$$
\begin{aligned}
& d(S x, S y, a) \\
& \leq q \psi(\max \{d(J x, I y, a), d(J x, S x, a), d(I y, S y, a), \\
& \left.\left.\quad \frac{d(J x, S y, a)}{2}, \frac{d(I y, S x, a)}{2}\right\}\right),
\end{aligned}
$$

where $0<q<1$ and $\psi \in \Psi$. If one of $S(X), I(X)$ and $J(X)$ is complete, then $S$ and $I, S$ and $J$ have an unique point of coincidence. Further, $S$ is one to one mapping, then $S, I, J$ have an unique point of coincidence.

Proof The proof is similar to that of Theorem 2.5. So we will omit it.

\section{REFERENCES}

[1] H. S. Yang and D. S. Xiong, "A Common Fixed Point Theorem on P-Metric Spaces," Journal of Yunnan Normal University (Science Edition), Vol. 21, No. 1, 2001, pp. 9-12.

[2] S. L. Singh, "Some Contractive Type Principles on 2Metric Spaces and Applications", Mathematics Seminar Notes (Kobe University), Vol. 7, No. 1, 1979, pp. 1-11.
[3] Y. J. Piao and Y. F. Jin, "Unique Common Fixed Point Theorem for a Family of Contractive Type Non-Commuting Selfmaps in 2-Metric Spaces," Journal of Yanbian University (Science Edition), Vol. 32, No. 1, 2006, pp. 13.

[4] Y. J. Piao, "A Family of Quasi-Contractive Type NonCommutative Self-Maps Having an Unique Common Fixed Point in 2-Metric Spaces," Journal of Heilongjiang University (Science Edition), Vol. 23, No. 5, 2006, pp. 655-657.

[5] Y. J. Piao, "Unique Common Fixed Point for a Family of Self-Maps with Same Type Contractive Condition in 2-Metric Spaces," Analysis in Theory and Applications, Vol. 24, No. 4, 2008, pp. 316-320. doi:10.1007/s10496-008-0316-9

[6] Y. J. Piao, "Unique Common Fixed Point for a Family of Self-Maps with Same Quasi-Contractive Type Condition in 2-Metric Space," Journal of Nanjing University (Mathematical Biquarterly), Vol. 27, No. 1, 2010, pp. 82-87.

[7] Y. J. Piao, "Uniqueness of Common Fixed Point for a Family of Mappings with $\phi$-Contractive Condition in 2Metric Space", Applied Mathematics, Vol. 3, 2012, pp. 73-77. doi:10.4236/am.2012.31012

[8] Y. J. Piao and Y. F. Jin, "New Unique Common Fixed Point Results for Four Mappings with $\Phi$-Contractive Type in 2-Metric Spaces," Applied Mathematics, Vol. 3, 2012, pp. 734-737. doi:10.4236/am.2012.37108

[9] M. Abbas and G. Jungck, "Common Fixed Point Results for Noncommuting Mappings without Continuity in Cone Metric Spaces," Journal of Mathematical Analysis and Applications, Vol. 341, No. 1, 2008, pp. 416-420. doi:10.1016/j.jmaa.2007.09.070

[10] Y. Han and S. Y. Xu, "New Common Fixed Point Results for Four Maps on Cone Metric Spaces," Applied Mathematics, Vol. 2, 2011, pp. 1114-1118. doi:10.4236/am.2011.29153

[11] C. D. Bari and P. Vetro, " $\Phi-$ Pairs and Common Fixed Points in Cone Metric Spaces," Rendicontidel Circolo Matematico Palermo, Vol. 57, 2008, pp. 279-285. 